\title{
Review of Agent-Based Systems in the Manufacturing Section
}

\author{
Georgios Andreadis ${ }^{1, *}$, K.-D. Bouzakis ${ }^{1}$, P. Klazoglou ${ }^{2}$, K.Niwtaki ${ }^{2}$ \\ ${ }^{1}$ Mechanical Engineering Dept, Aristotle University, Thessaloniki, 54124, Greece \\ ${ }^{2}$ Dept. of Informatics Aristotle University, Thessaloniki, 54124, Greece \\ *Corresponding Author: andreadi@eng.auth.gr
}

Copyright (C) 2014 Horizon Research Publishing All rights reserved.

\begin{abstract}
The manufacturing sector is considered an important field to the industry. Its products help manufacturers to achieve more control with less labor and more output with less cost. Current technology and especially Artificial Intelligence effectively increase productivity and reduce the cost of operations, which is the key to commercial success. The main representatives of information technology in the industrial applications are the agents. They provide an efficient way to design and implement engineering environments and the agents' technology is recognized as a new approach for the $\mathrm{CAD} / \mathrm{CAPP} / \mathrm{CAM}$ systems. This paper provides a view of the current agent technology in forenamed systems and constitutes an attempt to a) categorize multi-agent systems according to the coordination mode among the agents and $b$ ) classify the agents that take action on them, depending on their functions.
\end{abstract}

Keywords Agents, Multi-agent Systems, CAD/CAM, Manufacturing

\section{Introduction}

In the history of information technology, the first calculating systems were isolated entities that communicated only with person-operators while recently these systems have been linked and characterized by intelligence [1]. The tendency for linking systems, independent operation, representation of profits and making decisions during persons or systems interaction, led to the establishment of a new field of information technology, the agents and at extension the multi-agent systems. Agents are usually responsible for the information flow in a system, in order to increase the competition and companies' efficiency. They deal with the integration of the existing systems in order to resolve problems concerned to business manufacturing facilities and automated control of industrial unions.

This paper is structured as follows. Section 1.1 investigates the features of the agents and multi-agent systems, while a brief view of both the design and the manufacturing stages and their application software are described in section 1.2 and 1.3. Section 2 introduces reader to the categorization of multi-agent systems architectures and discusses the principles of centralized and decentralized architectures. Section 3 presents a classification of the agents in centralized systems in design and manufacturing stages. Respectively, section 4 classifies the agents that belong to decentralized systems into design and manufacturing stages. Finally, section 5 summarizes the basic problems which might be solved by the agents.

\subsection{Agents and Multi-Agents Systems}

Agent technology is an autonomous scientific field which during previous years has exceptional research activity [2]. As in every technology, the agents posses a variety of special skills and that is why they are useful for distributed, unstructured and decentralized architectures that change usually and thus are complicated. The term "agent" [3] is characterized by the meaning of autonomy which means self-activity for its objective achievements. A question with uncertain answers is:" Are agents intelligent?" This question is not easy to be answered. Although this article's purpose is not to answer it, we will briefly present the features that outline an intelligent agent. An intelligent agent has to react on environment changes and allocate capabilities and characteristics, such as social ability.

Agents interact, collaborate, coordinate and negotiate in a system that was designed and implemented as a multi-agent system. A multi-agent architecture is based on cooperative intelligent entities and has been developed for the integration of design, manufacturing and shop-floor control activities. There are two types of multi-agent coordination which are described further down. Agents communicate by exchanging messages. Messages type, pattern, semantics and the way of interconnection is defined by each communication protocol.

\subsection{Design and Manufacturing}

Engineering aims at quick product turnover and better 
balanced products by integrating various issues concerning the design and manufacturing phases. The process of product manufacturing is complex enough and thus it is necessary to be distinguished for production increase and quality improvement. The main stages of producing parts are the design and the manufacturing stages.

As fig. 1 depicts, design process consists of the following main phases: (a) the conceptual design (b) the functional design and (c) the representation [4]. The most abstract phase is the first one as it regards the product's initial concept. The second phase concludes how the technical specifications can be fulfilled while the final stage constitutes of the analytic geometric representation of the product. The abstract solution is impressed via linear drawings or three dimension models, using CAD software. The manufacturing process includes optimization procedures using CAM and CAD/CAPP software.

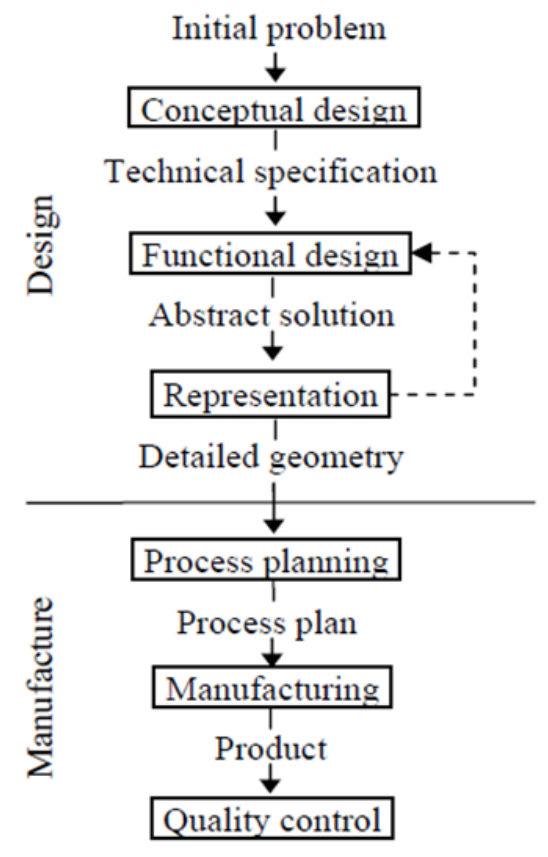

Figure 1. Design and Manufacturing steps [4]

\subsection{Application Software}

Since design and manufacturing phase is not similar, the software used for each one has different features. The design and manufacturing stage that is aided by the computers is named Computer Integrated Manufacturing (CIM). As far as the stage of solution representation is concerned, various software systems have been developed and allow the rapid creation of linear drawings or 3-D models (Computer Aided Design-CAD). CAD is capable of creating rapid linear systems, drawings and three dimension models. CAM involves tool design, Computer aided Process Planning (CAPP), planning of machine-tools, implementation and quality control (CAQC) (fig. 2) and CAM programs are capable of exporting from the 3-D products models to manufacture the analytical directives of the stages. Agents have enhanced intelligent design and manufacturing technologies at the aforementioned systems and have been applied in various manufacturing stages.

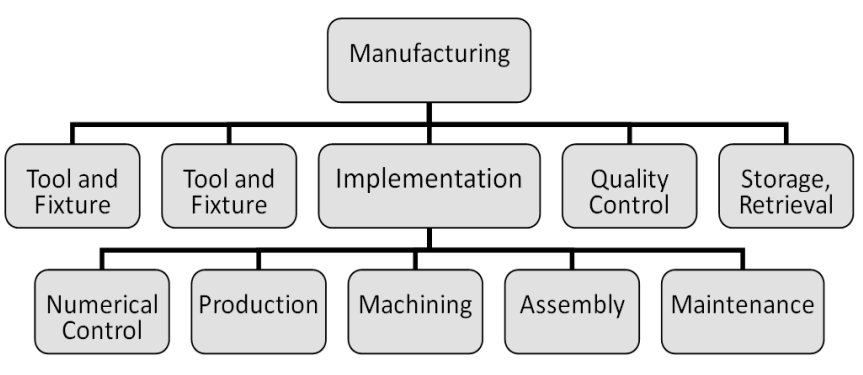

Figure 2. Manufacturing Stages

In the past, many reviews related to the use of artificial intelligence in manufacturing, have been presented through various conferences and journal publications $[5,6]$.

\section{Categorization of Multi-Agent System Architectures}

This section demonstrates the main approaches of multi-agent architectures, according to the coordination way. As fig. 3 depicts, the main coordination types are: a) centralized multi-agent coordination (or coordinator agents), in which one central agent undertakes the collection of partial plans from the agents, combines them in a plan and solves possible conflicts and b) decentralized multi-agent coordination (or autonomous agents), in which the agents are not controlled by a central agent although they communicate with each other for the creation of their plans and the solution of possible arguments. Next section refers to centralized architectures aspect while section 2.2 analyses decentralized architectures or autonomous agents.

Although there are several aspects that we can consider in order to classify CAD, CAPP and CAM multi-agent systems, this review develops a classification scheme, based on interaction characteristics, which has the following advantages:

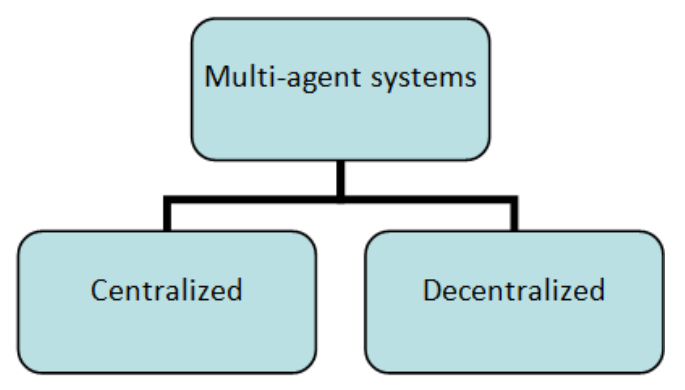

Figure 3. Multi agent systems categories

- This categorization is easily applied as it depends on the existence, or not, of a coordinator agent. For instance, much multi-agent architecture includes 
facilitator or mediator agents and thus these systems are clearly centralized. In contrast, classification based on agent function is more ambiguous, as an agent may handle operations from several engineering stages.

- Communication among the agents has played an increasingly important role in multi-agent architectures and is a key research area in multi-agent systems. Analysis of the interaction mode may highlight a variety of potential problems and suggest possible ways of resolution. Therefore, the specific classification focuses on agent coordination.

\subsection{Centralized Architectures}

In centralized architectures an agent coordinator manages the other agents. Coordinator is an agent that manages other agents and directs information flow among the other agents. Each agent has to contact the coordinator agent in order to communicate with each other. Fig. 4 shows a centralized architecture in which the coordinator agents facilitate coordination among the agents. Moreover, we consider an architecture centralized whether there is a central coordinator that handles coordinator's communication or not. The main representatives of agent coordinator are the mediator and the facilitator. In the rest of this paper, we call centralized a multi-agent system that owns a facilitator agent, a mediator or an agent that undertakes the management and the cooperation of the other agents. Their brief description follows at sections 2.2.1 and 2.2.2 respectively.

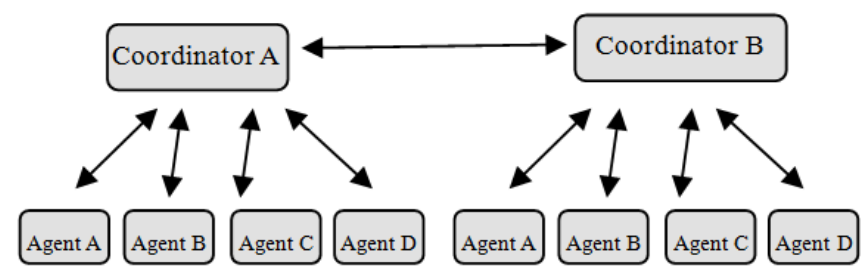

Figure 4. Centralized architecture

\subsubsection{Agent Facilitator}

A facilitator is a program that coordinates the communication of agents. The facilitator provides a reliable level of communication in the network and is responsible of routing messages between agents, based on their content, and regulating the control of multi-agent activities. As Fig 4 depicts, the agents interact via the facilitators (coordinators), which translate the tool - specific knowledge from and to a standard knowledge interchange language. In these systems, the communication and the coordination are accomplished among agents and facilitators or among facilitators but not directly via the agents.

\subsubsection{Agent Mediator}

The mediator takes on low level decisions only for crucial situations. The mediator may include behaviors of mediation that may focus in high policies in order to break decisions deadlock. The actions of mediation are performance-directed behaviors. The mediator uses mechanisms of brokerage and recruitment for the achievement of communication in order to find relative agents for the establishment of collaborative subsystems. Lastly, the mediator's responsibilities involve message interpretation, tasks decomposition and the benefit of the treatment's time for each new subtask.

\subsection{Decentralized architectures}

Initially, we define an autonomous agent. Although there are several definitions in the research literature, according to [7] autonomous is an agent which concentrates the following attributes: (a) it is not controlled or directed of a software agent or person, (b) it can communicate/interact immediately with other agents in the system or in other systems, (c) it has knowledge for other agents and their environment and (d) it devotes its own plans and an associated total of motives.

According to Maes [8], the emphasis in autonomous agent architectures is on more direct coupling of perception and action, distributeness and decentralization, dynamic interaction with the environment and intrinsic mechanisms to cope with resource limitation and incomplete knowledge. Fig. 5 shows that the agents are independent and communicate directly with each other. These agents are capable of communicating with agents that belong to other networks.

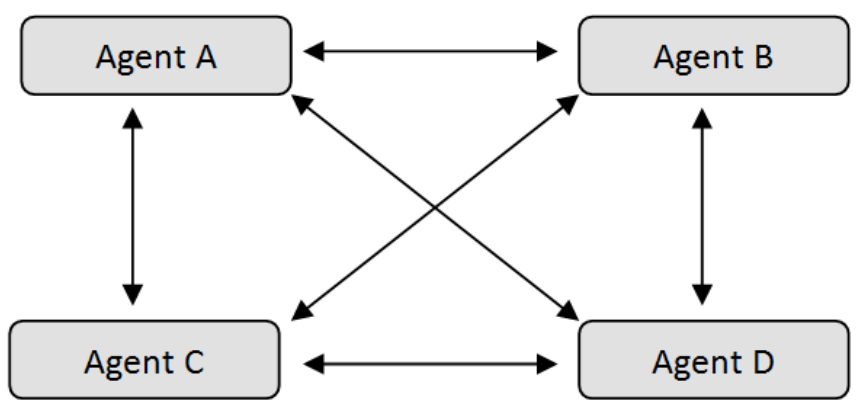

Figure 5. Decentralized architecture

Decentralized multi-agent architectures involve operations associated with preliminary design, manufacturing planning, distributed surface machining system, operation of a machine-tool and many other operations. Generally, classical planning and scheduling mechanisms have been substituted by task decomposition within a community of autonomous agents associated with their subtasks without a central control mechanism. Decentralized systems work on how a community of agents can make decisions and perform tasks within a manufacturing company or across companies and communication problems among agents with the lack of central control.

A large number of projects related with Agent-Based Systems in the Manufacturing Section have been presented through various conferences and journal publications [9-17]. 


\section{Conclusion}

This study is based on the use of agents in the mechanical applications $[18,28]$. The bibliography that was used comes from both engineering and informatics section. Before we result in the conclusions, it is judged necessary the enumeration of the reasons that led us to the use agents in engineering. First problem constitutes the lack of interoperability among heterogeneous software systems and tools. Secondly, current computing environments, such as material databases and resource databases, tend to be distributed because of new needs in industry. In our days, it is obvious the tendency for replacing rigid centralized and hierarchical systems with distributed architectures, such as client-server and peer to peer architectures. Moreover, $\mathrm{CAD} / \mathrm{CAM}$ systems are not linked with the aforementioned systems and this constitutes one more problem because industrial needs demand the integration of software systems, such as tools and existing databases with CAD/CAM system. Thirdly, manufacturing cost has been increased because of the use of sub-optimal process parameters. Last, but not least important is the lack of web-based collaboration between the process planning activity and the process parameter optimization activity. Web-based collaboration can improve the procedure as well as offer important services, such as video conferencing and electronic data interchange.

Thereupon, the agents provide functionality in the existing software systems. The agents facilitate the design and the manufacturing procedures of machining parts. Generally, their use consists of the accomplishment of individual operations and via their interaction they amplify systems functionality.

Finally, this study results in the further numbered conclusions:

1) Firstly, we have to note the existence of a similar agent in several systems, which are used for similar operations. Both design agent and service provider agent belong to this category.

2) As far as naming agents, there are agents with common actions but different names in several systems. For example, facilitator and blackboard agent facilitate communication operations. In reverse, there are agents with the same name but different functions. It should be highlighted that some agents take their name from their systems (for example, DFx agent belongs to DFx system that supports distributed design).

3) The existence of an agent in a centralized system does not exclude its operation at a decentralized architecture. For example, design agent operates both at centralized and decentralized systems. Exceptions are coordinator agents that are used in centralized systems only.

4) The design stage includes fewer agents than the manufacturing stage. This is predictable since the design of mechanical parts is not so automated and also, design data are incomplete, ambiguous and unorganized.

5) Several multi-agent systems are characterized by agents' hierarchy, since some agents have more profits and responsibilities in a system. For example, mediator agent in an assembly process planning framework assigns business tasks to process planners and mediates complications when their results do not coincide. So, it is responsible for other agents that belong to lower levels

6) Scientific society focuses to communication and coordination issues, routing and exchanging messages. The communication means for knowledge sharing technology for software interoperability are FIPA ACL, KQML and CORBA.

7) Important issues that have to be more developed are network security, data integrity and private data modification. As for security, there are cipher techniques and certification authorities that provide secure communication and data transfer among entities.

\section{REFERENCES}

[1] Wooldridge M., 2002, An introduction to Multi Agent Systems, John Wiley \& Sons, LTD, ((ISBN-10)0-471-49691-X(pbk)

[2] Vlaxavas I., Kefala s P., Vasiliadis N., Kokkoras F., Sakelariou H., 2006, Artificial Intelligence, Giourdas (ISBN: 960-387-431-0)

[3] Wallace A., Boldyreff C., 1999, Agents and Agent Based Design Approaches to Engineering Design and Manufacturing, University of Durham, UK

[4] Bouzakis K., Andreadis G., Vakali A., Katsonis P., 2006, An Agent-Based Software for Mechanical Design and Manufacture, Aristotle University of Thessaloniki, Greece

[5] Shen W., Hao Q., Yoon, Norrie D., 2006, Applications of agent-based systems in intelligent manufacturing: An update review, Advanced Engineering Informatics 20 (2006) 415-431

[6] Shen W., Norrie D., 1999, Agent-Based Systems for Intelligent Manufacturing: A State-of-the-Art Survey, Knowledge and Information Systems, an International Journal, 1(2), 129-156

[7] Shen W., Norrie D., 1997, Facilitators, Mediators or Autonomous Agents, Division of Manufacturing Engineering, the University of Calgary 2500 University Dr. NW, Calgary, Alberta, Canada T2N $1 \mathrm{~N} 4$

[8] Maes P., 1990, Designing Autonomous Agents, MIT Press, Cambridge, MA/ Elsevier, Amsterdam

[9] Jian Xun Wang, Ming Xi Tang, Lin Nan Song, Shou Qiang Jiang. Design and implementation of an agent-based collaborative product design system. Computers in Industry 2009;60(7):520-535

[10] Hong Liu, Ming Xi Tang, John Hamilton Frazer. Supporting 
evolution in a multi-agent cooperative design environment. Advances in Engineering Software 2002;33(6):319-328

[11] B Feijó, J Bento. A logic-based environment for reactive agents in intelligent CAD systems. Advances in Engineering Software 1998;29(10):825-832

[12] W.D. Fang, Ming Xi Tang, John Hamilton Frazer. Constructing an intelligent collaborative design environment with distributed agents. Proceedings of the 8th International Conference on Computer Supported Cooperative Work in Design 2004;1:329-335

[13] B. Feijó, P. C. R. Gomes, S. Scheer, J. Bento. Online algorithms supporting emergence in distributed CAD systems. Advances in Engineering Software 2001;32(10-11):779-787

[14] Weiming Shen, Qi Hao, Shuying Wang, Yinsheng Li, Hamada Ghenniwa. An agent-based service-oriented integration architecture for collaborative intelligent manufacturing. Robotics and Computer-Integrated Manufacturing 2007;23(3): 315-325

[15] Hong Liu, Ming Xi Tang. Evolutionary design in a multi-agent design environment. Applied Soft Computing 2006;6(2): 207-220

[16] Jiaqing Yu, Jianzhong Cha, Yiping Lu, Wensheng Xu, M. Sobolewski, 2010, A CAE-integrated distributed collaborative design system for finite element analysis of complex product based on SOOA, Advances in Engineering Software 41 (2010) 590-603

[17] Shaw C. Feng, Heli Helaakoski, Harri Haapasalo, Janne Kipina. Software agents-enabled systems coalition for integrated manufacturing processes and supply chain management. International Journal of Manufacturing Technology and Management 2007;11(2):157-173

[18] Alvares A., Ferreira J., 2006, Development of a system internet based collaborative cad capp cam in a context of e-manufacturing, ABCM Symposium Series in Mechatronics, Vol.2, pp.547-554

[19] Hao Q., Shen W., Park S., Lee J., Zhang Z., Shin B., 2005, An Agent-Based E-engineering Services Framework for Engineering Design and Optimization, 305-343
[20] Hao Q., Shen W, Zhang Z., Park S., Lee J., 2006, Agent-based collaborative product design engineering: An industrial case study, Computers in Industry 57 (2006) 26-38

[21] Riha A., Pechoucek M., Vokrinek J., Marik V., 2005, ExPlanTech: Exploitation of Agent-Based Technology in Production Planning, Gerstner Laboratory, Department of Cybernetics Czech Technical University in Prague

[22] Neaga I., 1998, Using information technology in manufacturing engineering, Technical University Department of Manufacturing Engineering, 9th DAAAM International Symposium, pp. 335-336, Romania

[23] Sang Hoon Kong, Sang Do Noh, Young-Gem Han, Gibom Kim, Kyo I1 Le, 2003, The Agent-based Collaborative Framework for Assembly Process Planning

[24] DAI-Kaiyu WANG-Yinglin ZHANG-Shensheng ZHANG Jingyi, 2002, Research on DFx Evaluation System for Distributed Design Based on Multi-Agent, Proceedings of the 2nd international Workshop on Autonomous Decentralized System

[25] Campbell M., Cagan J., Kotovsky K., 1999, A-Design: An Agent-Based Approach to Conceptual Design in a Dynamic Environment, Research in Engineering Design (1999)11:172-192

[26] Yang A., Braunschweig B., Fraga E., Guessoum Z., Marquardt W., Nadjemi O., Paend D., Pinol D., Roux P., Sama S., Serra M., Stalker I., 2008, A multi-agent system to facilitate component-based process modeling and design, Computers and Chemical Engineering 32 (2008) 2290-2305

[27] He, N., D. Z. Zhang, and Q. Li. "Agent-based hierarchical production planning and scheduling in make-to-order manufacturing system." International Journal of Production Economics (2013).

[28] Owliya, Mohammad, et al. "A new agents-based model for dynamic job allocation in manufacturing shopfloors." Systems Journal, IEEE 6.2 (2012): 353-361. 